\title{
CUMULATIVE MEASURE OF CORRELATION FOR MULTIPARTITE QUANTUM STATES
}

\author{
ANDRÉ L. FONSECA DE OLIVEIRA and EFRAIN BUKSMAN
}

JESÚS GARCÍA LÓPEZ DE LACALLE

\begin{abstract}
The present article proposes a measure of correlation for multiqubit mixed states. The measure is defined recursively, accumulating the correlation of the subspaces, making it simple to calculate without the use of regression. Unlike usual measures, the proposed measure is continuous additive and reflects the dimensionality of the state space, allowing to compare states with different dimensions. Examples show that the measure can signal critical points (CPs) in the analysis of Quantum Phase Transitions (QPTs) in Heisenberg models.
\end{abstract}

Keywords: Quantum information; quantum correlation measure; Heisenberg model; quantum phase transitions.

PACS numbers: 03.65.Ud, 03.67.Mn, 64.70.Tg, 05.30.RT, 75.10.Jm

\section{Introduction}

Quantum correlations are very significant in quantum information tasks. ${ }^{1}$ For this reason, quantifying correlations is one of the most important problem in quantum information theory. ${ }^{2}$

Many quantum correlation measures have already been proposed: entanglement of formation, geometric entanglement, quantum discord, etc. ${ }^{3-5}$ Although the first type of quantum correlation studied was bipartite, today both entanglement and quantum discord have several generalizations for the multipartite case. ${ }^{6-8}$ The relevance of multipartite correlation applies for quantum information processing, ${ }^{9,10}$ 
for speeding up quantum algorithms ${ }^{11}$ and for the study of many-body systems. ${ }^{12}$ Unfortunately, most of these measures can only be analytically determined for a few qubits; or require the calculation of nonlinear regressions, which limits the possibilities of studying more complex systems.

In Sec. 2 a total correlation measure for multiqubit mixed states is defined recursively, accumulating the correlation of the subspaces, and hence, making it simple to calculate without the use of regression. The proposed measure has the property of being a continuous additive measure that reflects the dimensionality of the state. Appendix A presents proofs for the measure properties.

In many-body physics analysis, correlation plays a fundamental role in quantum phase transition (QPT). In highly correlated systems, QPT is due to quantum fluctuations. ${ }^{13}$ The behavior of quantum discord and entanglement measures in the analysis of QPT in several Heisenberg spin chain models, shows that correlation measures can be used to detect critical points (CPs) ${ }^{14-16}$ As shown in Sec. 3, the proposed measure serves as an indicator of CPs in QPT, even in low noise environments.

Conclusions and proposed future work are presented in Sec. 4.

\section{Cumulative Correlation Measure}

\subsection{Definition}

In this section a definition for a new cumulative measure of correlation for multiqubit states is introduced. The proposed correlation computes total (quantum and classical) correlation.

Definition 1. Given a multiqubit state $\rho$, the cumulative correlation measure (CCM) is defined as

$$
C(\rho)=\min _{\{k\}}\left[2^{N-2} D\left(\rho, \rho_{A_{k}} \otimes \rho_{B_{k}}\right)+C\left(\rho_{A_{k}}\right)+C\left(\rho_{B_{k}}\right)\right]
$$

where $D(\cdot, \cdot)$ denotes a quantum distance, and $k$ is an index for an element $\left(\rho_{A_{k}}, \rho_{B_{k}}\right)$ in the set of all possible bipartitions of the state.

The proposed measure starts computing the sum of the distance between state $\rho$ and the product of its reduced matrices $\left(\rho_{A_{k}}\right.$ and $\rho_{B_{k}}$ ), weighted by a dimensional factor $\left(2^{N-2}\right)$, accumulating the CCM of each part, $C\left(\rho_{A_{k}}\right)$ and $C\left(\rho_{B_{k}}\right)$. The recursion stops for one qubit states $\left(C\left(\rho_{1 q}\right)=0\right)$. The final result is the minimum among all partitions.

Remark 1. In order to achieve the properties listed in the next subsection, the distance $D(\cdot, \cdot)$ used must have the following features:

(1) Invariance. $D\left(U \rho U^{\dagger}, U \sigma U^{\dagger}\right)=D(\rho, \sigma)$, where $U$ is a unitary operator.

(2) Contractivity. $D(\varepsilon(\rho), \varepsilon(\sigma)) \leq D(\rho, \sigma)$, where $\varepsilon(\sigma)$ are quantum local operations $\left(\varepsilon=\varepsilon^{1} \otimes \cdots \otimes \varepsilon^{N}\right)$. 
(3) Monotonicity. $D\left(\rho_{A}, \sigma_{A}\right) \leq D\left(\rho_{A B}, \sigma_{A B}\right)$.

Remark 2. In this paper the relative entropy is used

$$
D_{\mathrm{RE}}(\rho, \sigma) \equiv S(\rho \| \sigma)=\operatorname{Tr} \rho(\log \rho-\log \sigma)
$$

as the distance in definition (1). Though not a distance, given the symmetric property failure, ${ }^{17}$ it is always used in the order defined in (2). Relative entropy satisfies the aforementioned properties. ${ }^{1}$ Because in the proposed measure $\sigma=\rho_{A_{k}} \otimes \rho_{B_{k}}$, the relative entropy is equal to the mutual information. ${ }^{18}$

\subsection{Properties of $C C M$}

In the last years some conditions that multiqubit correlation measures should satisfy have been discussed. ${ }^{2,18,19}$ Here properties satisfied by CCM are shown, whereas proofs are presented in Appendix A.

Property 1. CCM must be positive or zero.

$$
C(\rho) \geq 0 .
$$

Property 2. Any product multiqubit state $\rho=\rho^{1} \otimes \cdots \otimes \rho^{N}$ has no correlation.

$$
C\left(\rho^{1} \otimes \cdots \otimes \rho^{N}\right)=0 \text {. }
$$

Property 3. CCM is invariant under local unitary transformations $\left(U=U^{1} \otimes \cdots \otimes\right.$ $\left.U^{N}\right)$.

$$
C\left(U \rho U^{\dagger}\right)=C(\rho)
$$

Property 4. CCM value is not affected in a system $\rho$ increased by locally noncorrelated auxiliary subsystems.

$$
C(\rho \otimes \sigma)=C(\rho),
$$

where $\sigma=\sigma^{1} \otimes \cdots \otimes \sigma^{K}$.

Property 5. Quantum local operations do not increase CCM.

$$
C(\varepsilon(\rho)) \leq C(\rho)
$$

where $\varepsilon=\varepsilon^{1} \otimes \cdots \otimes \varepsilon^{N}$.

The above properties are commonly accepted for a good correlation measure. ${ }^{20}$ Besides these, CCM also has the following properties.

Property 6. CCM is an additive measure.

$$
C(\phi \otimes \varphi)=C(\rho)+C(\varphi) .
$$

Property 7. CCM reflects the dimensionality of the state space. Two conditions express this: 
(1) Maximum correlation measure is non-decreasing with dimensionality.

$$
\max _{\left\{\mathcal{H}_{\mathcal{M}}\right\}} C\left(\rho^{M}\right) \leq \max _{\left\{\mathcal{H}_{\mathcal{N}}\right\}} C\left(\rho^{N}\right),
$$

where $M$ and $N$ are the dimension of the states, and $M \leq N$.

(2) For GHZ states ${ }^{21}$ the correlation measure increases with dimension.

$$
C\left(\rho_{\mathrm{GHZ}}\right)<C\left(\rho_{\mathrm{GHZ}}\right)
$$

where $M \leq N$.

Besides these properties is important to note that the proposed measure is continuous.

\subsection{CCM for $\mathrm{GHZ}$ states}

Using decimal notation to simplify the description of canonical base vectors (e.g., $|101\rangle=|5\rangle), N$ qubits GHZ states can be described as

$$
\begin{aligned}
|\mathrm{GHZ}\rangle_{\mathrm{N}} & =\frac{\sqrt{2}}{2}(|0 \cdots 0\rangle+|1 \cdots 1\rangle)=\frac{\sqrt{2}}{2}\left(|0\rangle+\left|2^{N}-1\right\rangle\right), \\
\rho_{\mathrm{GHZ}} & =|\mathrm{GHZ}\rangle\left\langle\left.\mathrm{GHZ}\right|_{N} .\right.
\end{aligned}
$$

For GHZ states, all the bipartite reduced matrices are of type

$$
\begin{aligned}
& \rho_{A_{k}}=\frac{1}{2}\left(|0\rangle\left\langle 0|+| 2^{N_{A k}}-1\right\rangle\left\langle 2^{N_{A k}}-1\right|\right), \\
& \rho_{B_{k}}=\frac{1}{2}\left(|0\rangle\left\langle 0|+| 2^{N_{B k}}-1\right\rangle\left\langle 2^{N_{B k}}-1\right|\right),
\end{aligned}
$$

where $N=N_{A k}+N_{B k}$. The product state in Eq. (1) has the form

$$
\begin{aligned}
\rho_{A_{k}} \otimes \rho_{B_{k}}= & \frac{1}{4}\left(|0\rangle\left\langle 0|+| 2^{N}-1\right\rangle\left\langle 2^{N}-1\right|\right. \\
& \left.+\cdots|m\rangle\left\langle m|+| 2^{N}-1-m\right\rangle\left\langle 2^{N}-1-m\right|\right),
\end{aligned}
$$

where $m \in\left\{1, \ldots, 2^{N-1}-1\right\}$. Essentially, they are all the same state (except for a change in the qubits). Therefore, all the distances between $\rho_{\mathrm{GHZ}}$ and $\rho_{A_{k}} \otimes \rho_{B_{k}}$ are equal, and are considered normalized (independent of the number of qubits $N$ ), i.e.,

$$
\begin{gathered}
D_{\mathrm{GHZ}} \equiv D\left(\rho_{\mathrm{GHZ}}, \rho_{A_{k}} \otimes \rho_{B_{k}}\right)=1, \\
\Rightarrow C\left(\rho_{\mathrm{GHZ}}\right)=2^{N-2} D_{\mathrm{GHZ}}+\min _{\{k\}}\left[C\left(\rho_{A_{k}}\right)+C\left(\rho_{B_{k}}\right)\right] \\
=2^{N-2}+\min _{\{k\}}\left[C\left(\rho_{A_{k}}\right)+C\left(\rho_{B_{k}}\right)\right] .
\end{gathered}
$$

The reduced matrices of $\rho_{A_{k}}$ are of the same type as (13) (as are $\rho_{B_{k}}$ ). Then, regardless of the dimension, the normalized distances between $\mathrm{A}$ and any of the Kronecker products of its reduced matrices are all the same, denoted here as $d$. 
Considering that the minimization for all partitions occurs (due to a power of 2 factor) for partitions with $N / 2$ qubits ( $N$ even), or with $(N+1) / 2$ and $(N-1) / 2$ qubits ( $N$ odd), the values of CCM for GHZ states can be computed with the following algorithm:

$$
\begin{aligned}
F(x, 2) & =x \\
F(x, 3) & =2 x+d \\
N \geq 4(\text { even }): F(x, N) & =2^{N-2} x+2 F\left(d, \frac{N}{2}\right), \\
N \geq 5(\text { odd }): F(x, N) & =2^{N-2} x+F\left(d, \frac{N-1}{2}\right)+F\left(d, \frac{N+1}{2}\right), \\
C\left(\rho_{\mathrm{GHZ}_{N}}\right) & =F(1, N),
\end{aligned}
$$

where $\rho_{\mathrm{GHZ}_{N}}$ is an $N$ qubit GHZ state. When the distance used is the relative entropy $\left(D_{\mathrm{RE}}\right)$, the constant $d$ equals $1 / 2$. Table 1 shows the proposed measure for the first nine GHZ states.

For example, in $\mathcal{H}_{4}$ the pure states

$$
\begin{aligned}
\rho_{1}=\left|\psi_{1}\right\rangle\left\langle\psi_{1}|, \quad| \psi_{1}\right\rangle & =\left|\mathrm{GHZ}_{2}\right\rangle \otimes\left|\mathrm{GHZ}_{2}\right\rangle \\
& =\frac{1}{2}(|0000\rangle+|0011\rangle+|1100\rangle+|1111\rangle), \\
\rho_{2}=\left|\psi_{2}\right\rangle\left\langle\psi_{2}|, \quad| \psi_{2}\right\rangle & =\left|\mathrm{GHZ}_{3}\right\rangle \otimes|0\rangle \\
& =\frac{\sqrt{2}}{2}(|0000\rangle+|1110\rangle), \\
\rho_{3}=\left|\psi_{3}\right\rangle\left\langle\psi_{3}|, \quad| \psi_{3}\right\rangle & =\left|\mathrm{GHZ}_{4}\right\rangle=\frac{\sqrt{2}}{2}(|0000\rangle+|1111\rangle)
\end{aligned}
$$

have as results $C\left(\rho_{1}\right)=2, C\left(\rho_{2}\right)=2.5$ and $C\left(\rho_{3}\right)=5$. In the first two states $\left(\rho_{1}\right.$ and $\rho_{2}$ ) the additive property of the measure is explicit. This example shows the influence of large correlated subspaces in high dimension quantum spaces. The larger the dimension of a correlated subspace, the greater the measure of correlation.

Table 1. CCM values for GHZ states GHZ.

\begin{tabular}{ccc}
\hline Number of qubits & CCM & CCM with $D_{\mathrm{RE}}$ \\
\hline 2 & 1 & 1 \\
3 & $2+\mathrm{d}$ & 2.5 \\
4 & $4+2 \mathrm{~d}$ & 5 \\
5 & $8+4 \mathrm{~d}$ & 10 \\
6 & $16+6 \mathrm{~d}$ & 19 \\
7 & $32+9 \mathrm{~d}$ & 36.5 \\
8 & $64+12 \mathrm{~d}$ & 70 \\
9 & $128+18 \mathrm{~d}$ & 137 \\
10 & $256+24 \mathrm{~d}$ & 268 \\
\hline
\end{tabular}




\section{CCM and Quantum Phase Transition}

In order to show the benefits of the proposed measure, some examples of strongly correlated spin chains, modeled by Heisenberg Hamiltonians, are studied. The generic spin $1 / 2$ Hamiltonian model $^{14}$ is given by

$$
H=-\sum_{i=1}^{N}\left(J_{x} \sigma_{i}^{x} \sigma_{i+1}^{x}+J_{y} \sigma_{i}^{y} \sigma_{i+1}^{y}+J_{z} \sigma_{i}^{z} \sigma_{i+1}^{z}+h \sigma_{i}^{z}\right)
$$

where $N$ is the number of spins, $\sigma^{x, y, z}$ are the Pauli matrices and the boundary condition $\sigma_{1}=\sigma_{N+1}$ is satisfied. In this paper only the case of interaction through nearest neighbors is analyzed.

Recently, several articles have reported detection of CPs in QPT models by different types of quantum correlations measures, i.e., entanglement, quantum discord and others. ${ }^{22-26}$ The selected examples, the Transverse Ising chain and the XXZ model, have the advantage that can be exactly solvable in one dimension, and the CPs are well known. ${ }^{27}$ The performance of CCM is studied for these models.

\subsection{The $X X Z$ model}

\subsubsection{Detection of critical points}

The Hamiltonian of the anisotropic XXZ model ${ }^{28,29}$ is given by

$$
H_{\mathrm{XXZ}}=-\frac{1}{2} \sum_{i=1}^{N}\left(\sigma_{i}^{x} \sigma_{i+1}^{x}+\sigma_{i}^{y} \sigma_{i+1}^{y}+\Delta \sigma_{i}^{z} \sigma_{i+1}^{z}\right)
$$

where $J_{x}=J_{y}=1 / 2$ and $h=0$, being $J_{z}=\Delta / 2$ the anisotropy parameter used in $(19)$.

The model exhibits three phases for the ground state: for $\Delta \rightarrow-\infty$ the chain is fully antiferromagnetic; for $-1<\Delta<1$ there is a gapless phase, and for $\Delta \rightarrow \infty$ has a fully polarized ferromagnet. These phases are separated by two CPs: at $\Delta=-1$ there is an infinite-order QPT, and a first-order QPT at $\Delta=1$.

Figure 1 shows the results of $\mathrm{CCM}$ and the total correlation measure defined in Ref. $30, \mathcal{T}_{V}$, is given by

$$
\mathcal{T}_{V}=S\left(\rho \| \rho_{1} \otimes \rho_{2} \cdots \rho_{N}\right)
$$

As observed, both measures have a discontinuity in $\Delta=1$, signaling a first-order $\mathrm{CP}$.

Figure 2 shows the details for $-1.5<\Delta<1$. While for $\mathcal{T}_{V}$ the value is constant, CCM presents a maximum for $\Delta=-1$ signaling the infinite-order CP. As expected, the maximum value grows with the number of spins in the chain (Fig. 3). 


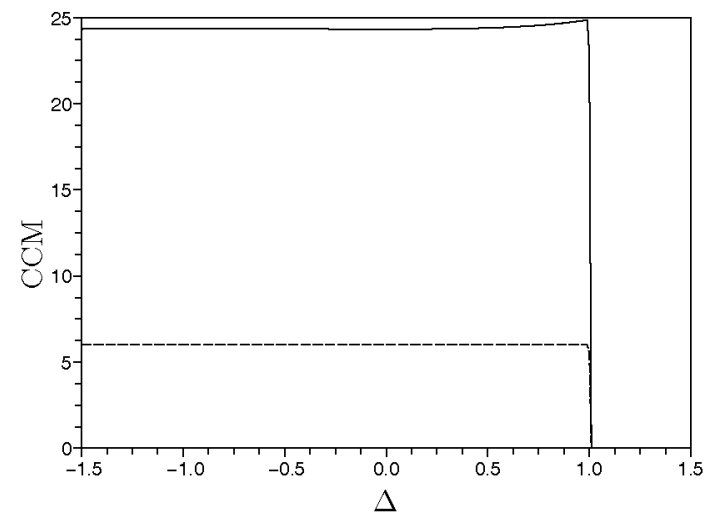

Fig. 1. Correlation (CCM and $\mathcal{T}_{V}$ ) of the grounded state and detection of CPs for XXZ chain of six spins. CCM (continuous line) and $\mathcal{T}_{V}$ (dashed line) for $-1.5<\Delta<1.5$. Both measures signaling the first-order CP at $\Delta=1$.

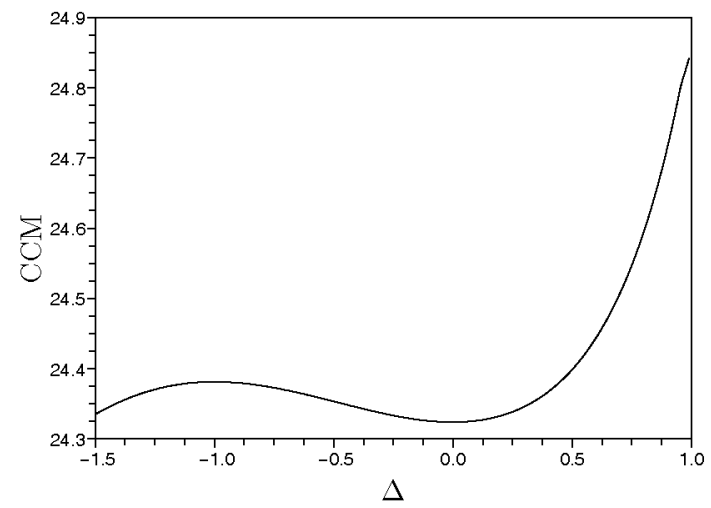

Fig. 2. Detail $(-1.5<\Delta<1)$ for CCM in the XXZ model. CCM can detect (maximum) the infinite-order CP at $\Delta=-1$.

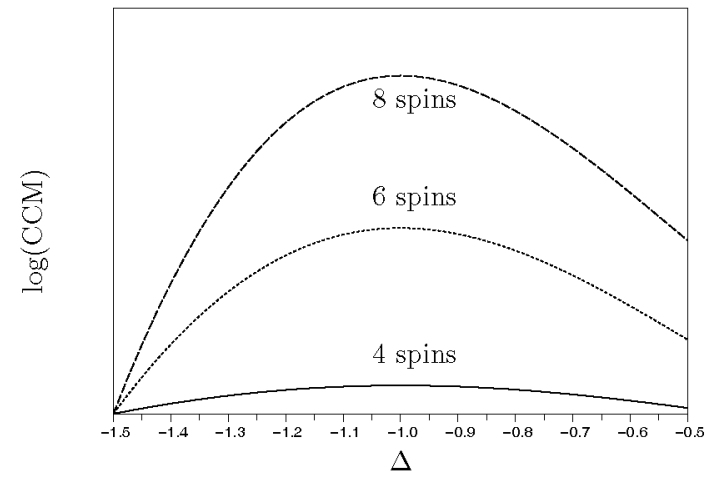

Fig. 3. CCM for XXZ chains of 4 (solid), 6 (dotted) and 8 (dashed) spins. In order to visualize the differences, the figure shows the logarithm of the measures. 


\subsubsection{Double chain QPT}

Unlike genuine measures, ${ }^{2,31} \mathrm{CCM}$ can signal the partial phase transition in a system. Consider the double chain Hamiltonian given by

$$
\begin{aligned}
H_{\mathrm{DXXz}}= & -\frac{1}{2} \sum_{i=1}^{N}\left(\sigma_{i}^{x} \sigma_{i+1}^{x}+\tau_{i}^{x} \tau_{i+1}^{x}+\sigma_{i}^{y} \sigma_{i+1}^{y}+\tau_{i}^{y} \tau_{i+1}^{y}+\cdots\right. \\
& \left.+\Delta \sigma_{i}^{z} \sigma_{i+1}^{z}+\lambda \tau_{i}^{z} \tau_{i+1}^{z}\right)
\end{aligned}
$$

where $\sigma^{x, y, z}$ and $\tau^{x, y, z}$ are the Pauli matrices of the two chains. This Hamiltonian represents a double independent XXZ model.

The results in Figs. 4 and 5 show that CCM can signal the partial phase transition in both subsystems.

\subsubsection{Open dynamics and QPT}

In the last years many articles deal with the behavior of the ground state of a spin chain considering the decoherence generated by the interaction with the environ-

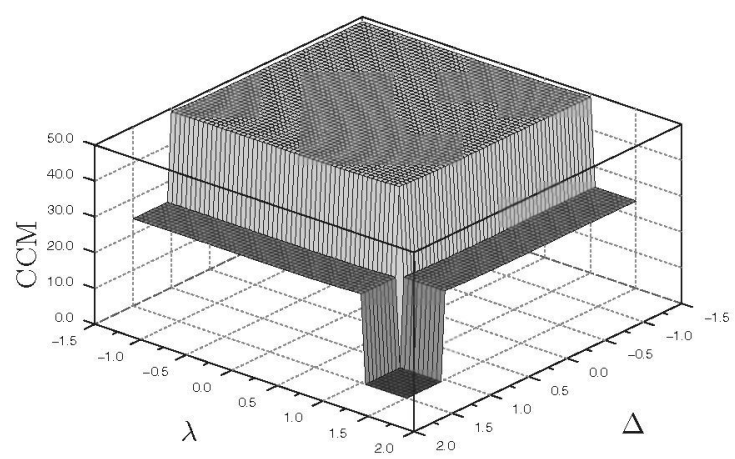

Fig. 4. Double chain QPT. Independent and simultaneous first-order CPs signaling.

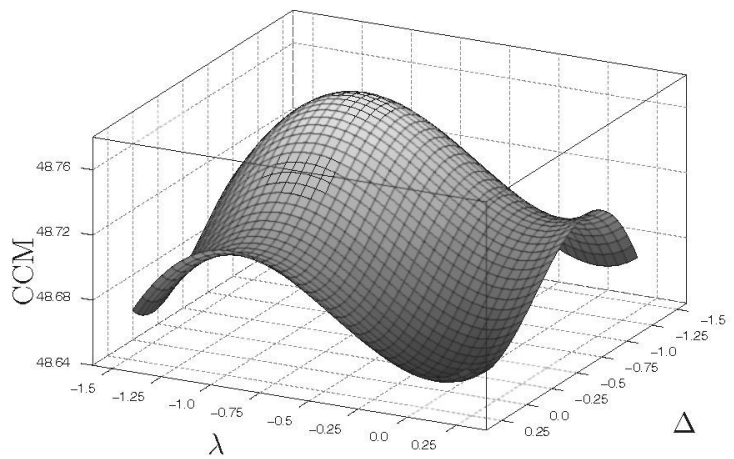

Fig. 5. Double chain QPT. Detail for the infinity-order double CP at $\Delta=-1$ and $\lambda=-1$. 
ment..$^{32-34}$ Real quantum systems are subject to interactions, because they can not be completely isolated. Then, it is of interest to know the behavior of fundamental states near CPs in the presence of noise caused by decoherence. As studied in Ref. 35, this effect can be achieved using Krauss operator representation, where the decoherence noise is typified by a set of operators $E_{i}$ that must hold $\sum_{i} E_{i} E_{i}^{\dagger}=I$. Then, decoherence can be expressed as

$$
\varepsilon(\rho)=\sum_{i} E_{i} \rho E_{i}^{\dagger}
$$

This example shows correlation evolution of XXZ fundamental states when amplitude damping noise operators are used,

$$
E_{0}=\left(\begin{array}{cc}
1 & 0 \\
0 & \sqrt{1-p}
\end{array}\right) \quad \text { and } \quad E_{1}=\left(\begin{array}{cc}
0 & 0 \\
0 & \sqrt{p}
\end{array}\right),
$$

to typify a dissipative interaction with the environment. ${ }^{1}$ In this case, the behavior is similar to the bipartite mutual information shown in Ref. 35. Figure 6 illustrates the evolution of the ground states near the infinity-order CPs $(-1.5<\Delta<1)$, considering a low noise approach $(0<p<0.04)$. It is interesting to note that the maximum is more pronounced with some amount of noise (Fig. 7 ).

\subsection{Transverse Ising model}

The Ising model has a different universality class of CP in comparison to the XXZ model. As in Ref. 28, the Hamiltonian of the transverse Ising model is given by (19) using $J_{x}=1, J_{y}=J_{z}=0$ and $h=\lambda$, resulting in

$$
H_{\text {Ising }}=-\sum_{i=1}^{N}\left(\sigma_{i}^{x} \sigma_{i+1}^{x}+\lambda \sigma_{i}^{z}\right) .
$$

For $\lambda=0$ all the spins point in the $x$-direction, while for $\lambda \rightarrow \infty$ all point in the $z$-direction. The ground state presents a CP at $\lambda=1$. This is signaled by an

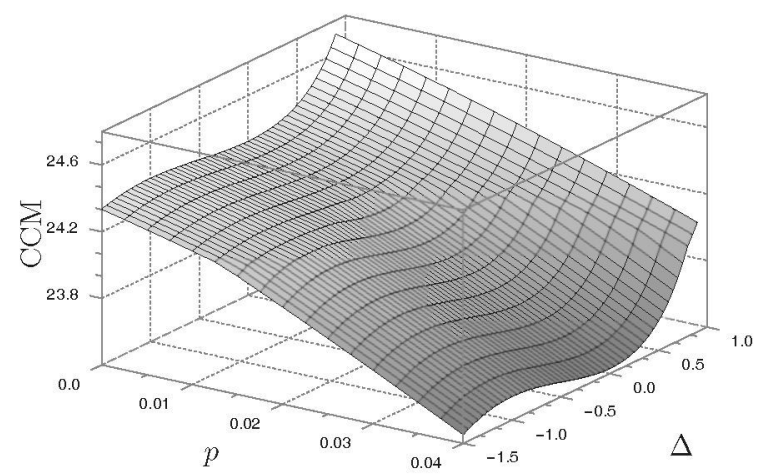

Fig. 6. CCM evolution of XXZ fundamental states in presence of noise. Evolution of fundamental states near the infinity-order CP. 


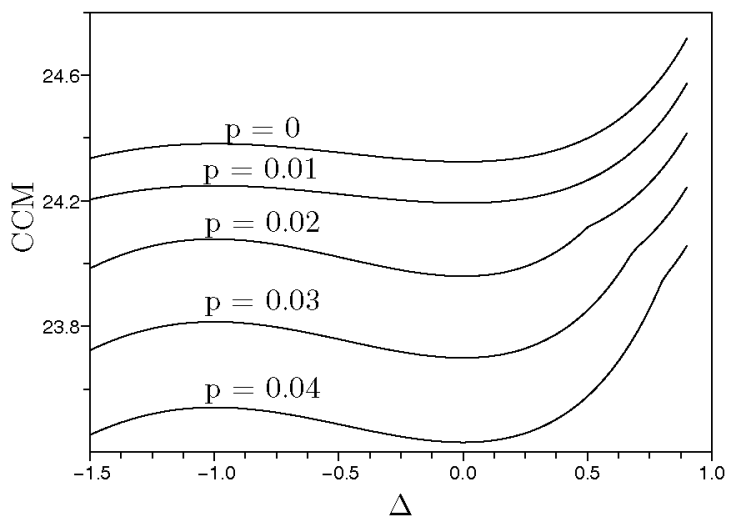

Fig. 7. CCM evolution of XXZ fundamental states in presence of noise. Contours for $p \in$ $\{0,0.01,0.02,0.03,0.04\}$.

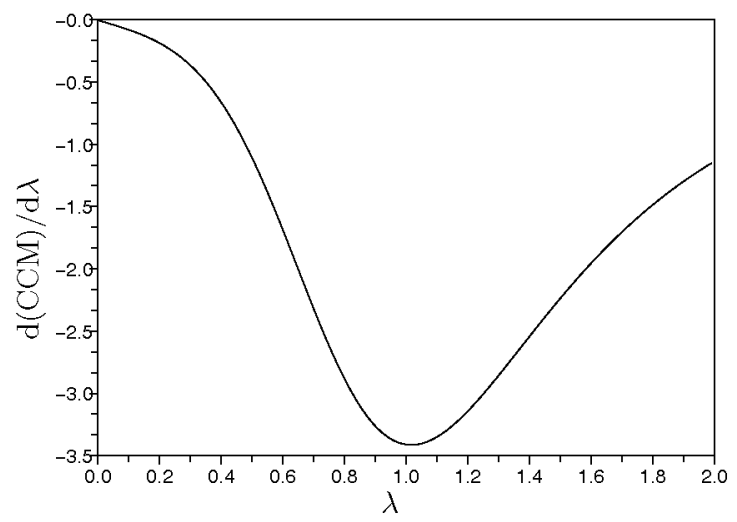

Fig. 8. Transverse Ising model. Derivative of CCM near the ground state.

inflexion point in the CCM curve (observed in Fig. 8 as a minimum in the derivative of CCM curve), similar to other measures of correlation. ${ }^{24,35-37}$

\section{Conclusions}

In summary, this paper proposes a total cumulative measure of correlation, CCM, which meets the expected properties for a multiqubit correlation, adding desirable features as additivity and dependence on the dimension of space.

Some results in the study of QPT have been illustrated. As in the case of quantum correlation (as quantum discord), CCM can be used to signal CPs. And, although the calculation grows exponentially with the dimension, nonlinear regression methods are not required. Another advantage is that for GHZ states the results can be known algebraically, facilitating comparison between different dimension systems. 
While in this article relative entropy is used as the distance of the algorithm, the proposal is actually a framework that can be defined according to the chosen distance, creating a relationship between each interpretation of correlation and each type of distance.

As future work, it is interesting to find good definitions for quantum correlation counterparts of this total correlation.

\section{Acknowledgments}

We thank the partial support from the Technology Development Program (PDT, Ministry of Education and Culture of Uruguay) through grant S/C/BE/55/23.

\section{Appendix A. Proof of CCM properties}

This section presents the proofs of CCM properties presented in subsection 2.2 .

\section{A.1. Property 1}

Proof. By distance definition, $D(\rho, \sigma) \geq 0$ for all $\rho$ and $\sigma$. Then, considering the recursive nature of the measure and that $C(\rho)=0$ for the ultimate decomposition (one qubit), $C(\rho) \geq 0$ for all $\rho$.

\section{A.2. Property 2}

Proof. This property is a direct consequence of the additivity (Property 6) proved below and that the correlation is zero for one-qubits states.

\section{A.3. Property 3}

Proof. Assuming a state $\rho^{\prime}=U \rho U^{\dagger}$, where $U=U^{1} \otimes \cdots \otimes U^{N}$ is a local unitary transformation, the CCM of the new state is

$$
C\left(\rho^{\prime}\right)=\min _{\{k\}}\left[2^{N-2} D\left(\rho^{\prime}, \rho_{A_{k}}^{\prime} \otimes \rho_{B_{k}}^{\prime}\right)+C\left(\rho_{A_{k}}^{\prime}\right)+C\left(\rho_{B_{k}}^{\prime}\right)\right]
$$

being $\rho_{A_{k}}^{\prime}$ and $\rho_{B_{k}}^{\prime}$ the $k$ bipartition of the transformed state. Using the same partition (and properly rearranging the qubits) and considering $U=U_{A_{k}} \otimes U_{B_{k}}$ we have that

$$
\rho_{A_{k}}^{\prime}=\operatorname{Tr}_{B_{k}}\left(\rho^{\prime}\right)=U_{A_{k}} \rho_{A_{k}} U_{A_{k}}^{\dagger}, \quad \rho_{B_{k}}^{\prime}=\operatorname{Tr}_{A_{k}}\left(\rho^{\prime}\right)=U_{B_{k}} \rho_{B_{k}} U_{B_{k}}^{\dagger},
$$

which implies that

$$
\rho_{A_{k}}^{\prime} \otimes \rho_{A_{k}}^{\prime}=U_{A_{k}} \rho_{A_{k}} U_{A_{k}}^{\dagger} \otimes U_{B_{k}} \rho_{B_{k}} U_{B_{k}}^{\dagger}=U_{k}\left(\rho_{A_{k}} \otimes \rho_{B_{k}}\right) U_{k}^{\dagger} .
$$

So, by the required invariance property of the distance

$$
\left.D\left(\rho^{\prime}, \rho_{A_{k}}^{\prime} \otimes \rho_{B_{k}}^{\prime}\right)=D\left(\rho_{k}, \rho_{A_{k}} \otimes \rho_{B_{k}}\right)\right], .
$$

By finite induction is easy to see that $C\left(\rho_{A_{k}}^{\prime}\right)=C\left(\rho_{A_{k}}\right)$ and $C\left(\rho_{B_{k}}^{\prime}\right)=C\left(\rho_{B_{k}}\right)$, so $\mathrm{CCM}$ is invariant under unitary local operations. 


\section{A.4. Property 4}

Proof. This property is a direct consequence of the Properties 2 and 6 .

\section{A.5. Property 5}

Proof. Consider local operations such that $\varepsilon(\rho), \varepsilon=\varepsilon^{1} \otimes \cdots \otimes \varepsilon^{N}$. The correlation of a state, resulted of applying a local operation $\varepsilon(\rho)$ is

$$
C(\varepsilon(\rho))=\min _{\{k\}}\left[2^{N-2} D\left(\varepsilon(\rho), \sigma_{A_{k}} \otimes \sigma_{B_{k}}\right)+C\left(\sigma_{A_{k}}\right)+C\left(\sigma_{B_{k}}\right)\right],
$$

where $\varepsilon=\varepsilon_{A_{k}} \otimes \varepsilon_{B_{k}}, \sigma_{A_{k}}=\operatorname{Tr}_{B_{k}}(\varepsilon(\rho))$ and $\sigma_{B_{k}}=\operatorname{Tr}_{A_{k}}(\varepsilon(\rho))$.

Using Stokes tensor ${ }^{38}$ is straightforward that $\sigma_{A_{k}} \otimes \sigma_{B_{k}}=\varepsilon\left(\rho_{A_{k}} \otimes \rho_{B_{k}}\right)$, where $\rho_{A_{k}}=\operatorname{Tr}_{B_{k}}(\rho)$ and $\rho_{B_{k}}=\operatorname{Tr}_{A_{k}}(\rho)$. Then, by contractivity (Sec. 2.1),

$$
\begin{aligned}
D\left(\varepsilon(\rho), \sigma_{A_{k}} \otimes \sigma_{B_{k}}\right) & =D\left(\varepsilon(\rho), \varepsilon\left(\rho_{A_{k}} \otimes \rho_{B_{k}}\right)\right) \\
& \leq D\left(\rho, \rho_{A_{k}} \otimes \rho_{B_{k}}\right) .
\end{aligned}
$$

Using this, we will prove by induction that the proposed measure is contractive, i.e.,

$$
C(\varepsilon(\rho)) \leq C(\rho)
$$

For two-qubit states, the property is a direct consequence of the distance between the state and the product of its reduced matrices.

Suppose now that for any state in a space of dimension less than or equal to $N-1$, the measure is contractive. Consider a multiqubit state $\rho$. With local operations, the correlation measure is

$$
C(\varepsilon(\rho))=\min _{\{k\}}\left[2^{N-2} D\left(\varepsilon(\rho), \sigma_{A_{k}} \otimes \sigma_{B_{k}}\right)+C\left(\sigma_{A_{k}}\right)+C\left(\sigma_{B_{k}}\right)\right] .
$$

For each partition $k$, by (A.5) we have that

$$
D\left(\varepsilon(\rho), \sigma_{A_{k}} \otimes \sigma_{B_{k}}\right) \leq D\left(\rho, \rho_{A_{k}} \otimes \rho_{B_{k}}\right) .
$$

As $\sigma_{A_{k}}$ and $\sigma_{B_{k}}$ have less than $N$ qubits,

$$
\begin{aligned}
& C\left(\sigma_{A_{k}}\right) \leq C\left(\rho_{A_{k}}\right), \\
& C\left(\sigma_{B_{k}}\right) \leq C\left(\rho_{B_{k}}\right) .
\end{aligned}
$$

Then for all $N$,

$$
C(\varepsilon(\rho)) \leq C(\rho) .
$$




\section{A.6. Property 6}

Proof. Now we prove the additivity of the proposed measure.

For a two-qubit product state the property is straightforward (unique partition),

$$
C(\rho)=C(\phi \otimes \varphi)=D(\rho, \phi \otimes \varphi)+C(\phi)+C(\varphi)=0 .
$$

In order to perform an induction let's assume that the property holds for all product states with less than $N$ qubits. Considering all possible partitions we have three cases:

(1) The full state $\rho$ is partitioned in the states $\phi$ and $\varphi$. For this particular partition, $k_{0}$, as $D(\rho, \phi \otimes \varphi)=0$, we have that

$$
C_{k_{0}}(\rho)=C(\phi)+C(\varphi) .
$$

(2) The used partition $k$ divides the state $\rho$ such that the first partial trace, $\rho_{A_{k}}$, includes the full state $\phi$ and some part of $\varphi$ (the same applies for the other partial trace). In this case we have

$$
\begin{aligned}
& \rho_{A_{k}}=\operatorname{Tr}_{B_{k}}(\phi \otimes \varphi)=\phi \otimes \varphi_{A_{k}^{\prime}}, \\
& \rho_{B_{k}}=\operatorname{Tr}_{A_{k}}(\phi \otimes \varphi)=\varphi_{B_{k}},
\end{aligned}
$$

where $A_{k}^{\prime}$ and $B_{k}$ is a partition in the $\mathcal{H}_{\varphi}$ subspace $\left(A_{k}\right.$ and $B_{k}$ is a partition in the hole $\mathcal{H}_{\varphi} \otimes \mathcal{H}_{\varphi}$ space). Then, as additivity holds for states with less than $N$ qubits,

$$
\begin{aligned}
C_{k}(\rho)= & 2^{N-2} D\left(\rho, \phi \otimes \varphi_{A_{k}^{\prime}} \otimes \varphi_{B_{k}}\right)+C(\phi)+\cdots \\
& +C\left(\varphi_{A_{k}^{\prime}}\right)+C\left(\varphi_{B_{k}}\right) .
\end{aligned}
$$

For state $\varphi$ we have that

$$
\begin{aligned}
C(\varphi) & =\min _{\{k\}}\left[C_{k}(\varphi)\right], \quad M<N \\
& =\min _{\{k\}}\left[2^{M-2} D\left(\varphi, \varphi A_{k}^{\prime} \otimes \varphi_{B_{k}}\right)+C\left(\varphi_{A_{k}^{\prime}}\right)+C\left(\varphi_{B_{k}}\right)\right] .
\end{aligned}
$$

By the monotonicity property (Sec. 2.1),

$$
D\left(\varphi, \varphi_{A_{k}^{\prime}} \otimes \varphi_{B_{k}}\right) \leq D\left(\rho, \phi \otimes \varphi_{A_{k}^{\prime}} \otimes \varphi_{B_{k}}\right) .
$$

Then,

$$
\Rightarrow C_{0}(\rho)=C(\varphi)+C(\phi) \leq C_{k}(\varphi)+C(\phi) \leq C_{k}(\rho), \quad \forall k .
$$

(3) The used partition $k$ divides the state $\rho$ such that both substates $\phi$ and $\varphi$ are partitioned, where $A_{k}^{\prime}$ and $B_{k}^{\prime}$, and $A_{k}^{\prime \prime}$ and $B_{k}^{\prime \prime}$ are partitions in the subspaces $\mathcal{H}_{\phi}$ and $\mathcal{H}_{\varphi}$, respectively. In this case

$$
\begin{aligned}
& \rho_{A_{k}}=\operatorname{Tr}_{B_{k}}(\phi \otimes \varphi)=\phi_{A_{k}^{\prime}} \otimes \varphi_{A_{k}^{\prime \prime}} \\
& \rho_{B_{k}}=\operatorname{Tr}_{A_{k}}(\phi \otimes \varphi)=\phi_{B_{k}^{\prime}} \otimes \varphi_{B_{k}^{\prime \prime}}
\end{aligned}
$$


and $C_{k}$ is

$$
\begin{aligned}
C_{k}(\rho)= & 2^{N-2} D\left(\rho,\left(\phi_{A_{k}^{\prime}} \otimes \varphi_{A_{k}^{\prime \prime}}\right) \otimes\left(\phi_{B_{k}^{\prime}} \otimes \varphi_{B_{k}^{\prime \prime}}\right)\right) \\
& +C\left(\phi_{q_{k}^{\prime}}\right)+C\left(\varphi_{q_{k}^{\prime \prime}}\right)+C\left(\phi_{\bar{q}_{k}^{\prime}}\right)+C\left(\varphi_{\bar{q}_{k}^{\prime \prime}}\right) .
\end{aligned}
$$

By a similar procedure to that used in (b) we have that $C_{0} \leq C_{k}, \forall k$. Then, for a multiqubit state the property holds, and by induction the measure is additive.

\section{A.7. Property 7}

Condition 1.

Proof. This property is straightforward. Suppose that $\rho_{M} \in \mathcal{H}_{M}$ is the state with maximum measure. In the space $\mathcal{H}_{N}, N=M+L$, we have the state $\phi_{N}=\rho_{M} \otimes \varphi_{L}$. By Property $6, C\left(\phi_{n}\right)=C\left(\rho_{M}\right)+C\left(\varphi_{L}\right) \geq C\left(\rho_{M}\right)$. Then the maximum correlation in $\mathcal{H}_{N}$ is greater or equal than $C\left(\rho_{M}\right)$.

Condition 2.

Proof. Proved in Sec. 2.3.

\section{References}

1. M. Nielsen and I. L. Chuang, Quantum Computation and Quantum Information (Cambridge University Press, UK, 2000).

2. C. H. Bennett et al., Phys. Rev. A 83, 012312 (2011).

3. H. Ollivier and W. H. Zurek, Phys. Rev. Lett. 88, 017901 (2001).

4. R. Horodecki et al., Rev. Mod. Phys. 81, 865 (2009).

5. K. Modi et al., Rev. Mod. Phys. 84, 1655 (2012).

6. T.-C. Wei and P. M. Goldbart, Phys. Rev. A 68, 042307 (2003).

7. M. Okrasa and Z. Walczak, Europhys. Lett. 80, 042302 (2011).

8. C. C. Rulli and M. S. Sarandy, Phys. Rev. A 84, 042109 (2011).

9. A. R. R. Carvalho, F. Mintert and A. Buchleitner, Phys. Rev. Lett. 93, 230501 (2004).

10. R. Gallego et al., Phys. Rev. Lett. 107, 210403 (2011).

11. D. Bruß and C. Macchiavello, Phys. Rev. A 83, 052313 (2011).

12. F. Verstraete, M. Popp and J. I. Cirac, Phys. Rev. Lett. 92, 027901 (2004).

13. S. Sachdev, Quantum Phase Transitions (John Wiley \& Sons Ltd, USA, 2007).

14. L. Amico, R. Fazio and A. O. V. Vedral, Rev. Mod. Phys. 80, 517 (2008).

15. C. C. Rulli and M. S. Sarandy, Phys. Rev. A 81, 032334 (2010).

16. F. Altintas and R. Eryigit, Ann. Phys. 327, 3084 (2012).

17. K. M. R. Audenaert and J. Eisert, J. Math. Phys. 46, 102104 (2005).

18. L. Henderson and V. Vedral, J. Phys. A: Math. Gen. 34, 6899 (2001).

19. A. Brodutch and K. Modi, Quantum Inform. Comput. 12, 0721 (2012).

20. D. L. Zhou, B. Zeng, Z. Xu and L. You, Phys. Rev. A 74, 052110 (2006).

21. D. M. Greenberger, M. Horne and A. Zeilinger, Bells Theorem, Quantum Theory and Conceptions of the Universe (Kluwer, Berlin, 1989).

22. A. Osterloh et al., Nature 416, 608 (2002). 
23. A. Anfossi et al., Phys. Rev. Lett. 95, 056402 (2005).

24. R. Dillenschneider, Phys. Rev. B 78, 224413 (2008).

25. J. Cui, J.-P. Cao and H. Fan, Phys. Rev. A 82, 022319 (2010).

26. J. Wilms et al., J. Stat. Mech. 1023 (2012).

27. J. R. Baxter, Exactly Solved Models in Statistical Mechanics (Academic Press, US, 1982).

28. M. S. Sarandy, Phys. Rev. A 80, 022108 (2009).

29. Y. Yao et al., Phys. Rev. A 86, 042102 (2012).

30. V. Vedral, Rev. Mod. Phys. 74, 197 (2002).

31. G. L. Giorgi et al., Phys. Rev. Lett. 107, 190501 (2011).

32. T. Werlang et al., Phys. Rev. Lett. 105, 095702 (2010).

33. J.-L. Guo and G.-L. Long, Eur. Phys. J. D 67, 53 (2013).

34. T. Werlang, G. A. P. Ribeiro and G. Rigolin, Int. J. Mod. Phys. B 27, 1345032 (2013).

35. A. K. Pal and I. Bose, Eur. Phys. J. B 85, 36 (2012).

36. A. Auerbach, Interacting Electrons and Quantum Magnetism (Springer-Verlag, Berlin, 1998).

37. T. J. Osborne and M. A. Nielsen, Phys. Rev. A 66, 032110 (2002).

38. G. Jaeger, Quantum Information: An Overview (Springer, New York, 2007). 